\title{
ANÁLISE DA RELAÇÃO ENTRE A HABILIDADE DE INTEGRAÇÃO VISUO-MOTORA E O DESEMPENHO ESCOLAR
}

\section{RELATIONSHIP ANALYSIS BETWEEN VISUAL-MOTOR INTEGRATION ABILITY AND ACADEMIC PERFORMANCE}

\author{
Débora Morais Pereira1, Rita de Cássia Tibério Araújo², \\ Ligia Maria Presumido Braccialli ${ }^{3}$
}

\section{RESUMO}

A integração visuo-motora é uma habilidade que envolve a percepção visual e a coordenação olho-mão. Alterações da habilidade perceptiva ou da capacidade de organização motora podem refletir em dificuldades de aprendizado da leitura, escrita e matemática. Este trabalho objetivou verificar a existência de relação entre a habilidade de integração visuo-motora e o desempenho escolar, bem como identificar se a habilidade de percepção visual correlaciona-se com o desempenho na atividade de leitura e se a habilidade de coordenação motora correlaciona-se com o desempenho na atividade de escrita. Participaram do estudo 77 alunos do $2^{\circ}$. ano do ensino fundamental de uma escola pública. Para a coleta de dados foram utilizados o Teste do Desenvolvimento da Integração Visuo-Motora e o Teste de Desempenho Escolar. Os resultados mostraram correlação significante entre a habilidade de integração visuo-motora e o desempenho em atividades de leitura $(r=0,230 ; p=0,044)$, escrita $(r=$ $0,244 ; p=0,033)$ e aritmética $(r=0,277 ; p=0,015)$; correlação significante entre a habilidade de percepção visual e leitura $(r=0,407 ; p=0)$ e correlação significante entre a habilidade motora e a escrita com letra cursiva $(p=0,039)$. Os resultados deste estudo estão em consonância com os da literatura, no que diz respeito à constatação da influência das habilidades de integração visuomotora, percepção visual e coordenação motora sobre o desempenho do aluno nas atividades escolares.

Palavras-chave: percepção visual; educação.

1 Terapeuta Ocupacional. Especialista em Desenvolvimento Infantil. Mestranda em Educação pela Faculdade de Filosofia e Ciências, UNESP, campus de Marília. Endereço: Rua Antonio Hideto Kobayashi, 47, Condomínio San Marino, Jardim América, São José dos Campos, São Paulo, CEP 12235-845. Telefone (12) 3019-4467/ 9144-1406. E-mail: debora_terapi@yahoo.com.br

2 Terapeuta Ocupacional. Doutora em Educação. Professora do Curso de Terapia Ocupacional da Faculdade de Filosofia e Ciências, UNESP, campus de Marília. Departamento de Educação Especial. Endereço: Av. Hygino Muzzi Filho, 737, Cidade Universitária, Marília, São Paulo, Cx Postal 181, CEP: 17.525-900, Telefone (14) 3402-1331. E-mail: ritac@marilia.unesp.br

3 Fisioterapeuta. Livre Docente em Reabilitação Física. Professora do Curso de Fisioterapia da Faculdade de Filosofia e Ciências, UNESP, campus de Marília. Departamento de Educação Especial. Endereço: Av. Hygino Muzzi Filho, 737, Cidade Universitária, Marília, São Paulo, Cx Postal 181, CEP: 17.525-900, Telefone (14) 3402-1331. E-mail: ligiapresumido@hotmail.com

Correspondência para: debora_terapi@yahoo.com.br

Como citar este artigo: Pereira DM, Araújo RCT, Braccialli LMP. Relationship analysis between visual-motor integration ability and academic performance. Journal of Human Growth and Development 2011; 21(3): 808-817.

Artigo submetido em 06.02.11, aceito em 20.08.11. 


\begin{abstract}
Visual-motor integration is a skill that involves visual perception and eye-hand coordination. Deficit in perceptual ability and motor organization capacity may reflect in reading, writing and arithmetic learning difficulties. This study aimed to verify the relationship between visual-motor integration ability and academic performance, as well, whether visual perception ability was correlated with reading performance and whether motor coordination ability was correlated with writing performance. Participants were 77 students in the 2nd. year of elementary education at a public school. To data collect were applied the Developmental Test of Visual-Motor Integration and the Academic Performance Test. The results showed a significant correlation between visual-motor integration ability and academic skills of reading $(r=0.230, p=0.044)$, writing $(r=0.244, p=0.033)$ and arithmetic $(r=0.277, p=0.015)$. In addition, was also identified significant correlation between visual perception and reading performance $(r=0.407, p=0)$ and between the motor coordination and cursive writing $(p=0.039)$. The results of this study are consistent with the literature, concerning the verification of visual-motor integration, visual perception and motor coordination abilities influence on the students performance in school activities.
\end{abstract}

Key words: visual perception; education.

\section{INTRODUÇÃO}

A integração visuo-motora é uma habilidade de interação harmoniosa entre os olhos e as mãos; envolve a percepção visual e a coordenação olho-mão; requer a capacidade de traduzir a percepção visual em função motora e é necessária para muitas atividades do ser humano como pegar um objeto e lançá-lo, escrever, desenhar, pintar, recortar, etc ${ }^{1,2}$.

A construção das coordenações visuo-motoras representa um papel essencial na aprendizagem da escrita ${ }^{3}$. Baixa habilidade perceptiva ou da capacidade de organização motora pode refletir em dificuldades de aprendizado da leitura, escrita e matemática ${ }^{4}$.

Baseando-se na idéia de que a inteligência e o conhecimento têm base sensório-motora e pensando na necessidade de se identificar crianças com dificuldades de aprendizagem, Keith E. Beery criou, nos anos 60, com a ajuda de Norman A. Buktenica, o Developmen-tal Test of Visual-Motor Integration (Teste do Desenvolvimento da Integração VisuoMotora), também conhecido como Beery $\mathrm{VMI}^{4}$. E, em 1990, Pinelli Júnior realizou sua adaptação para uso no Brasill.
Segundo os autores do Beery VMI, no processo do desenvolvimento, a criança pode ter as habilidades visuais e motoras, mas ser incapaz de coordenálas. Desta forma, esse teste pretende avaliar a maturação da integração visuomotora ${ }^{4}$, bem como distinguir um conjunto de desordens características de crianças com dificuldades de aprendizagem mediante reprodução de figuras geométricas ${ }^{5}$.

Este teste é relativamente independente frente a aspectos culturais, o que o torna válido para aplicação tanto nos Estados Unidos quanto em outros países e é particularmente sensível como preditor do desempenho de grupos socioeconômicos baixos ${ }^{4}$.

Visto que componentes cognitivos como a percepção visual e a integração visuo-motora estão envolvidos no processo de aprendizagem, a utilização de testes neuropsicológicos na área de aprendizagem apresenta-se como uma ferramenta importante para desvendar causas cognitivas que possam acarretar dificuldades de aprendizagem ${ }^{6}$.

Outro teste que também pode ser utilizado na área de aprendizagem como uma ferramenta importante para identi- 
ficar dificuldades de aprendizagem é o Teste de Desempenho Escolar (TDE).

O TDE é um instrumento desenvolvido e padronizado no Brasil, concebido para avaliação das capacidades fundamentais para o desempenho escolar (escrita, leitura e aritmética) em escolares de $1^{\mathrm{a}}$ a $6^{\mathrm{a}}$ séries do Ensino Fundamental. Possui propriedades psicométricas adequadas no que diz respeito à sua confiabilidade interna?.

Considerando os propósitos do Beery VMI e a abrangência de aplicação do TDE, este trabalho objetivou verificar se existe correlação entre o desempenho na habilidade de integração visuo-motora e o desempenho escolar (leitura, escrita e aritmética), bem como identificar se a habilidade de percepção visual está correlacionada com o desempenho em atividade de leitura e se a habilidade de coordenação motora está correlacionada com o desempenho em atividade de escrita.

\section{MÉTODO}

Participaram do estudo 77 alunos do $2^{\circ}$ ano do ensino fundamental de uma escola pública do interior paulista, sendo 40 meninas e 37 meninos. A idade das crianças variou de 6 anos e 9 meses a 8 anos e 4 meses, com média de 7 anos e 6 meses. Para a coleta de dados foram utilizados dois instrumentos: o Teste do Desenvolvimento da Integração Visuomotora (Beery VMI) e o Teste de Desempenho Escolar.

O Beery VMI é um teste utilizado para avaliar a habilidade de integração visuo-motora. Consiste na reprodução de 24 figuras geométricas dispostas em ordem crescente de dificuldade. É composto de dois testes suplementares, o de Percepção Visual, do inglês Visual Perceptual (VP) e o de Coordenação Motora, do inglês Motor Coordination (MC) os quais foram adicionados na revisão realizada em 1997 no intuito de proporcionar recurso para avaliar estatisticamente as contribuições visual e motora para a performance na integração visuo-motora ${ }^{4}$. Este teste foi adaptado para uso no Brasil por Pinelli Junior (1990), mas não há padronização da pontuação por faixa etária para crianças brasileiras. Essa situação, entretanto, não se configura como um obstáculo metodológico neste estudo, pois não foi objetivo deste trabalho verificar se a pontuação obtida pelos escolares está dentro do esperado para sua faixa etária.

O TDE é um instrumento composto por três subtestes: escrita, aritmética e leitura. Busca oferecer uma avaliação objetiva das capacidades fundamentais para o desempenho escolar, indicando, de maneira abrangente, quais as áreas da aprendizagem escolar que estão preservadas ou prejudicadas no examinan$\mathrm{do}^{7}$. Neste teste, o uso de letra cursiva ou de forma dependeu da iniciativa da criança.

O trabalho foi encaminhado ao Comitê de Ética em Pesquisa da Faculdade de Filosofia e Ciências, UNESP - Marília, e aprovado sob o parecer $n^{\circ}$. 1923/2010. Os responsáveis pelos participantes assinaram o Termo de Consentimento Livre e Esclarecido para a participação voluntária na pesquisa.

Os testes foram aplicados durante o horário escolar, mas em sala reservada especificamente para este fim. O Beery VMI foi aplicado coletivamente em grupos de 5 ou 6 alunos e o TDE individualmente. Os testes suplementares do Beery VMI foram aplicados individualmente. A aplicação dos testes foi realizada em dias diferentes para não cansar os alunos, e a ordem da aplicação dos testes (Beery VMI e TDE) variou entre os participantes. A coleta de dados foi realizada diariamente, conforme disponibilidade dos professores, durante um período de aproximadamente dois meses.

Após a análise dos dados coletados, foi redigido e entregue aos responsáveis um relatório com o desempenho obtido pelas crianças nos testes aplicados. No caso dos alunos que apresentaram atraso importante na habilidade de integração visuo-motora, de percepção visual e/ ou coordenação motora foram fornecidas à escola e aos responsáveis, sugestões de atividades para auxiliar no desenvolvimento destas habilidades. 
A pontuação dos testes aplicados foi realizada conforme instruções contidas em seus manuais. Foram criados gráficos para melhor visualização dos dados e utilizou-se o programa MINITAB para a análise estatística dos dados. Para medir o grau de correlação entre o desempenho dos alunos no Beery VMI e no TDE foi utilizado o coeficiente de correlação de Pearson. Este teste também foi utilizado para verificar a existência de correlação entre percepção visual e leitura de palavras. Para verificar a possível relação entre a habilidade de coordenação motora e tipo de letra, foi utilizado o teste Two-sample T. Em toda a análise estatística foi adotado o nível de significância de 5\% $(\leq 0,05)$.

\section{RESULTADOS}

No histograma de pontuação no VMI (gráfico 1) encontram-se os resultados obtidos pelos 77 escolares nos testes de integração visuo-motora, percepção visual e coordenação motora. No teste de integração visuo-motora a pontuação variou de 10 a 25, sendo a média de 18 acertos. Na avaliação da percepção visual os alunos tiveram uma pontuação média de 21 , sendo 7 a menor pontuação e 21 a maior. E no teste de coordenação motora, a pontuação variou de 15 a 28, sendo 22 a média alcançada.

No histograma de pontuação no TDE (gráfico 2) verifica-se a pontuação alcançada nos subtestes de escrita, arit-

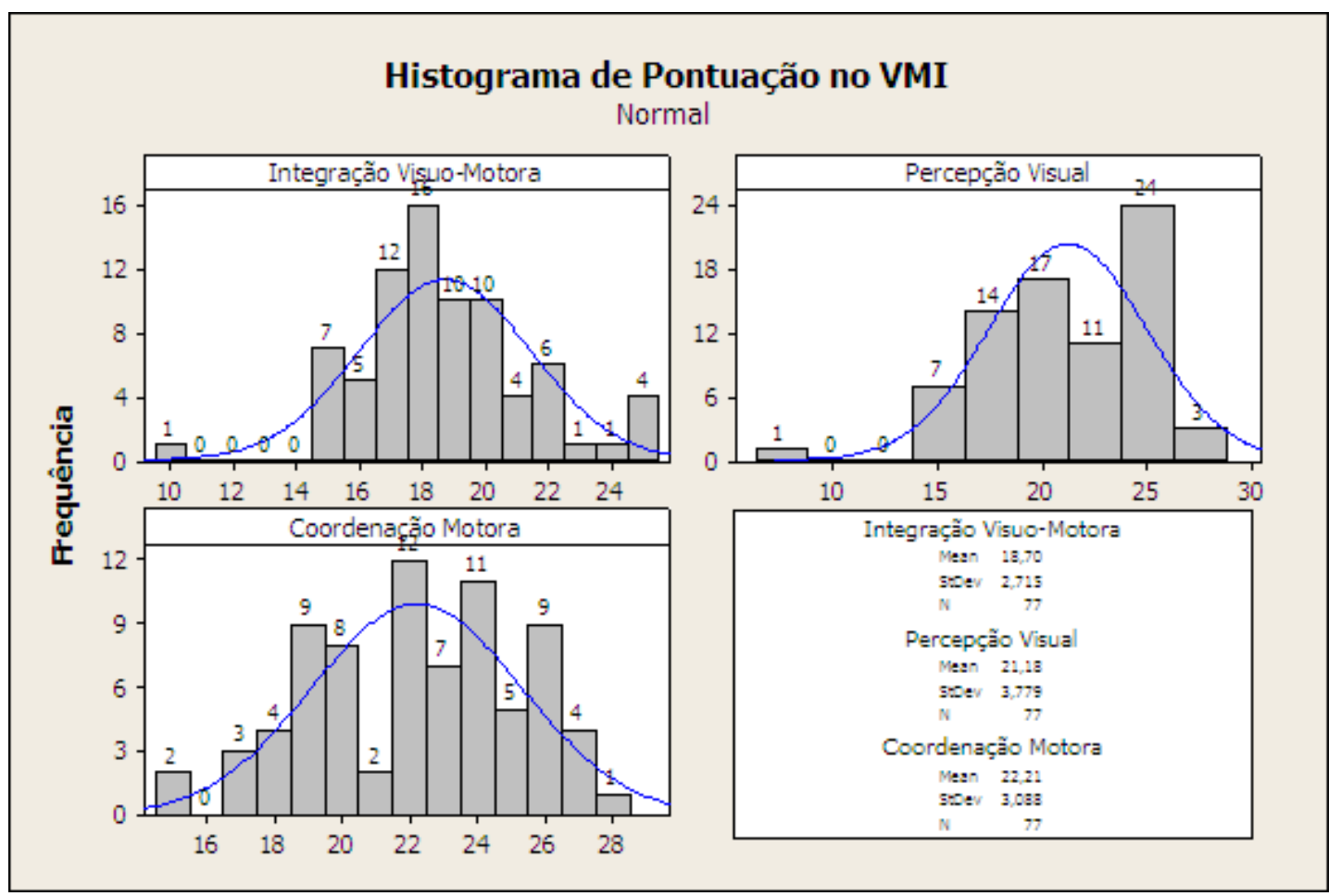

Gráfico 1: Histograma da pontuação obtida no VMI

mética e leitura. A média de acertos no subteste de escrita foi 13 , sendo a variação de zero a 28 acertos. Na avaliação de aritmética, a pontuação variou de zero a 14 , sendo a média de 8 pontos. E no subteste de leitura, houve uma variação de zero a 70, sendo a média de 46 pontos. Para verificar a correlação entre a ha- bilidade de integração visuomotora e as habilidades acadêmicas de escrita, aritmética e leitura foi aplicado o teste estatístico de correlação de Pearson. Os coeficientes de correlação de Person ( $r$ ) e o valor de " $p$ " obtidos podem ser verificados na Tabela 1, e a visualização da correlação positiva pode ser observada no Gráfico 3. 


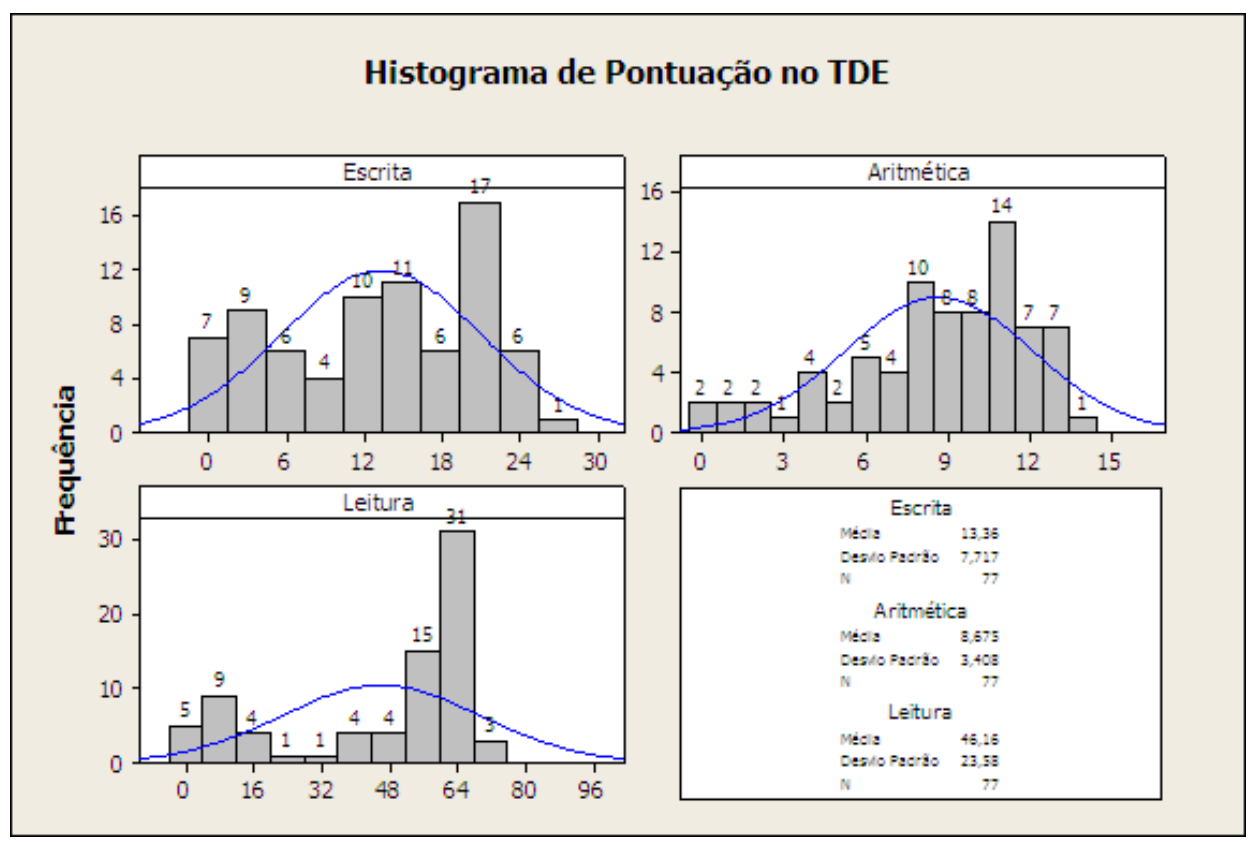

Gráfico 2: Histograma da pontuação obtida no Teste de Desempenho Escolar (TDE)

Tabela 1: Correlação de Pearson entre a habilidade de integração visuo-motora e as habilidades acadêmicas de escrita, aritmética e leitura

Coeficiente de correlação Valor de P de Pearson ( $r$ )

\begin{tabular}{llll}
\hline Integração Visuo-Motora & x Escrita & 0,244 & 0,033 \\
Integração Visuo-Motora & $\times$ Aritmética & 0,277 & 0,015 \\
Integração Visuo-Motora & $\times$ Leitura & 0,230 & 0,044 \\
\hline
\end{tabular}

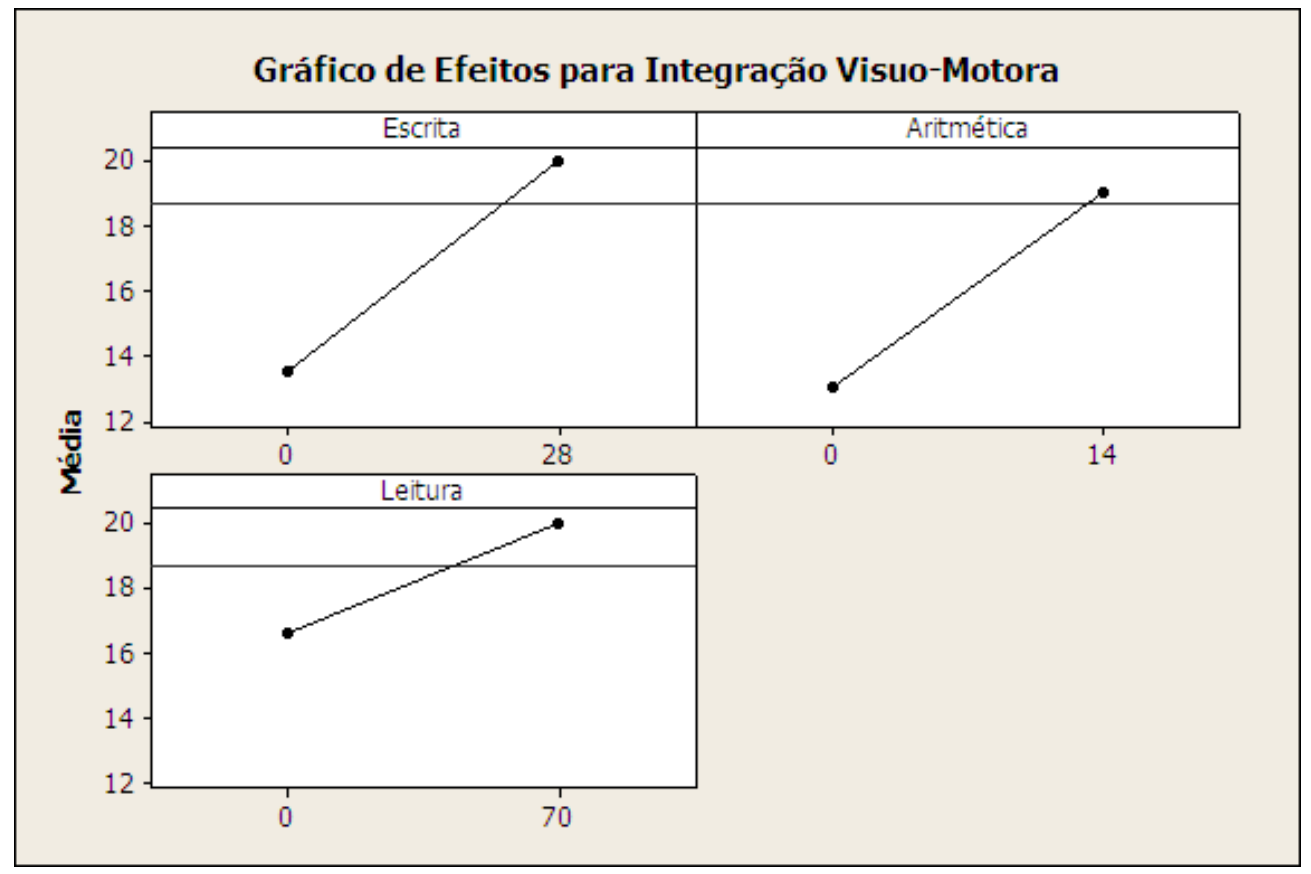

Gráfico 3: Correlação entre habilidade de integração visuo-motora e habilidades acadêmicas de escrita, aritmética e leitura. 
Também foi aplicado o teste de correlação de Pearson para verificar a existência de correlação entre o desempenho no teste de percepção visual e o desempenho no subteste de leitura. $O$ resultado obtido foi um coeficiente de correlação de Pearson de 0,407 e um valor de "p" igual a zero (0).

Para verificar a existência de correlação entre a habilidade de coordenação motora e a habilidade de escrever (com letra cursiva e de forma) foi aplicado o teste estatístico Two-Sample T. Este teste é usado para comparar as médias de duas amostras entre si e determinar se existe diferença estatística entre estas médias. $\mathrm{Na}$ Tabela 2, encontram-se a média e o desvio padrão do desempenho no teste de Coordenação Motora, segundo o tipo de letra (cursiva ou de forma), indicando-se também o valor de " $p$ " para a diferença encontrada entre os tipos de letras.

Tabela 2: Teste Two-sample T de diferenciação estatística entre o desempenho no teste de coordenação motora com letra de forma e com letra cursiva.

\begin{tabular}{cccc}
\hline Tipo de Letra & Número de sujeitos $(\mathbf{N})$ & Média & Desvio Padrão \\
\hline Cursiva & 25 & 23,32 & 3,33 \\
Forma & 52 & 21,67 & 2,85 \\
\hline
\end{tabular}

*Teste - T de diferença $=0$; Valor de $p=0,039$

Distribuição da Pontuação de Coordenação Motora por Tipo de Letra

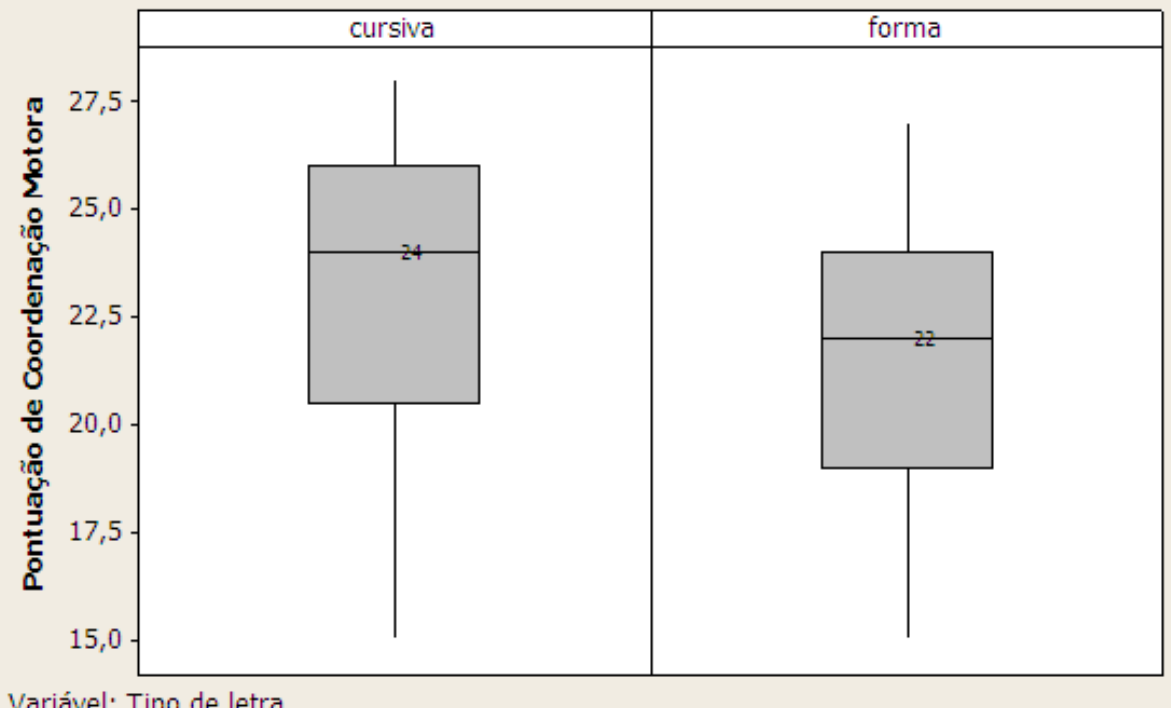

Variável: Tipo de letra

Gráfico 4: Distribuição da pontuação no teste de coordenação motora entre as letras cursiva e de forma.

O gráfico 4 permite a melhor visualização da correlação entre a pontuação no teste de coordenação motora e o tipo de letra (cursiva ou de forma).

\section{DISCUSSÃO}

Os resultados obtidos no teste estatístico de correlação de Pearson (Tabe- la 1) indicam que existe uma correlação significante entre a habilidade de integração visuo-motora e as habilidades acadêmicas avaliadas.

Na literatura estrangeira são encontrados diversos trabalhos que relacionaram a habilidade de integração visuomotora avaliada pelo Beery VMI com habilidades importantes para o sucesso acadêmico. Um exemplo é o trabalho de 
Kulp (1999) que também teve como objetivo examinar a relação entre a habilidade de integração visuo-motora e o desempenho acadêmico. Foram participantes do estudo 191 alunos de uma escola primária em Ohio, Estados Unidos, com faixa etária entre 7 e 9 anos. Os alunos do primeiro ano foram avaliados nas habilidades de integração visuomotora, leitura, escrita e matemática. Os alunos do segundo e do terceiro ano foram avaliados nessas habilidades e também em ortografia. Os resultados obtidos pelo autor citado mostraram que o desempenho no VMI relacionou-se significativamente com a avaliação dos professores em relação à leitura, escrita, matemática e ortografia ${ }^{8}$.

Outro trabalho que avaliou a habilidade de integração visuo-motora com - Beery VMI e a correlacionou com importante habilidade acadêmica foi conduzido por Daly, Kelley e Krauss (2003) com o objetivo de examinar a relação entre o desempenho no teste VMI e a legibilidade da escrita em 54 crianças que freqüentavam creche. Neste estudo, os alunos que conseguiram copiar as primeiras nove formas do VMI apresentaram um desempenho significativamente meIhor do que os alunos que não conseguiram copiar corretamente as mesmas nove formas. Este resultado levou os autores a concluírem que a habilidade de integração visuo-motora mostrou-se relacionada com a capacidade de copiar letras de forma legível ${ }^{9}$.

Nogueira, et al. (2007) também destacaram a importância da habilidade de integração visuo-motora, bem como da coordenação motora fina, no desempenho escolar. Estes pesquisadores, numa tentativa de diminuir o fracasso escolar nas séries iniciais através do resgate das habilidades psicomotoras, realizaram um estudo com 20 alunos, de 4 a 8 anos, a fim de detectar as defasagens psicomotoras das crianças, ressaltar a importância dos movimentos corporais na aquisição da escrita e identificar os múltiplos aspectos psicomotores que influenciam o processo de alfabetização. Neste estudo foram avaliadas, entre outras habilidades, a coordenação motora fina e visuo-motora. Os resultados especificamente destas habilidades apontaram que apenas $50 \%$ das crianças avaliadas conseguiram realizar todas as atividades que objetivavam avaliar estes aspectos, indicando, segundo os autores, uma dificuldade dos participantes para realizar atividades que exigiam o uso simultâneo das mãos e olhos (integração visuo-motora). Outra dificuldade bastante evidente esteve relacionada ao movimento de preensão: $60 \%$ das crianças seguraram incorretamente o lápis ou o hidrocor. Também foi percebido que as crianças, de uma maneira geral, apresentaram lentidão para registros do quadro e cópia, preensão incorreta do lápis e/ou da caneta, além de controle inadequado da pressão aplicada sobre o lápis/ caneta e borracha ${ }^{10}$.

Com relação à análise da relação entre a percepção visual e o desempenho em atividade de leitura, no presente estudo, o teste de correlação de Pearson mostrou correlação significante entre 0 desempenho obtido no teste de percepção visual e o obtido no subteste de leitura.

No que tange à análise da relação entre a coordenação motora e o desempenho em atividade de escrita, o valor de $p(0,039)$ obtido no teste estatístico Two Sample-T indicou diferença estatística significante entre o desempenho com letra cursiva e o desempenho com letra de forma $(p<0,05)$, podendo-se concluir, por meio das médias, que a iniciativa de escrever com letra cursiva está relacionada com a habilidade motora.

Não foram encontrados na literatura estudos específicos sobre a relação entre a variável percepção visual e a variável leitura nem entre a variável coordenação motora e a variável tipo de letra. No entanto, tem-se o trabalho de Oliveira, Lopes e Magalhães (2004) que descreveu o desempenho de crianças com 6 e 7 anos no Beery VMI e examinou a relação da pontuação neste teste com a acuidade do traçado e o nível de habilidade motora dessas crianças. Neste estudo o subteste visual apresentou correlação significante com o tempo gasto para a realização de atividade de cópia de tex- 
to e o teste motor apresentou correlação significante com o tempo de cópia e com a avaliação pela professora da qualidade da escrita. Segundo as autoras, os resultados obtidos deram suporte à relação entre o desempenho visuo-motor e a qualidade da escrita ${ }^{11}$.

Alterações no desenvolvimento da coordenação motora têm sido uma constante entre os escolares que apresentam problemas de aprendizagem. Segundo Capellini, Copede e Valle (2010), a literatura refere que ao menos $50 \%$ dos escolares com dificuldades de aprendizagem apresentam dificuldades de natureza motora. Preocupadas com tal prevalência, as autoras realizaram um estudo com o intuito de caracterizar o desempenho da função motora fina, sensorial e perceptiva em escolares com dislexia, distúrbio e dificuldades de aprendizagem e correlacionar estes achados à escrita destes escolares. Participaram deste estudo 80 escolares de $2^{\mathrm{a}}$ a 4 a série, de ambos os gêneros e com idade entre 7 e 11 anos. Desses alunos, 20 apresentavam diagnóstico interdisciplinar de dislexia (Grupo I), 20 de distúrbio de aprendizagem (Grupo II), 20 apresentavam dificuldades de aprendizagem (Grupo III) e os outros 20 não apresentavam tais dificuldades (Grupo IV). Estas crianças foram submetidas à avaliação da função motora fina (que é dividida em três partes: função motora fina, função motora sensorial e função motora perceptiva) e à análise da escrita por meio da aplicação da escala de disgrafia na avaliação da escrita de um bilhete sob ditado. Os resultados apontaram que a alteração motora esteve presente em escolares de todos os grupos. Quanto à classificação da função motora fina, sensorial e perceptiva, entre os escolares com dislexia, $20 \%$ apresentaram disfunção moderada, 50\% disfunção leve e 30\% não apresentaram disfunção; entres os escolares com distúrbio de aprendizagem, 95\% apresentaram disfunção leve e $5 \%$ não apresentaram disfunção; entre os escolares com dificuldades de aprendizagem, 90\% apresentaram disfunção leve e $10 \%$ não apresentaram disfunção e entre os escolares sem difi- culdades de aprendizagem, 55\% apresentaram disfunção leve e 45\% não apresentaram disfunção. Quanto à presença de disgrafia, esta foi verificada em $85 \%$ dos escolares com dislexia, $100 \%$ dos escolares com distúrbio de aprendizagem, $45 \%$ dos escolares com dificuldades de aprendizagem e em $15 \%$ dos escolares sem dificuldades de aprendizagem ${ }^{12}$.

Segundo Feder e Majnemer (2007) para uma adequada qualidade da escrita, isto é, uma boa caligrafia, são necessários, entre outras coisas, adequado controle motor fino e da integração visuomotora, planejamento motor, propriocepção, percepção visual, atenção sustentada e consciência sensorial dos dedos. Ainda segundo as autoras, o prejuízo nessas habilidades pode resultar em caligrafia ilegível e comprometer o desempenho acadêmico da criança ${ }^{13}$.

De maneira geral, o processo de aprendizagem envolve sistemas e habilidades diversas, inclusive motoras ${ }^{14}$. A aprendizagem da leitura e da escrita é um processo complexo e difícil para as crianças em fase inicial de alfabetização, engloba tanto fatores biológicos quanto sociais e exige habilidades cognitivas, lingüísticas e motoras ${ }^{15,16}$.

As crianças com dificuldades de aprendizagem demonstram dificuldades perceptivas quanto à identificação, discriminação e interpretação de estímulos que parecem repercutir em dificuldades na leitura, escrita e cálculo ${ }^{17}$. Quanto aos fatores sociais que parecem interferir no aprendizado escolar são citados na literatura o ambiente escolar e o ambiente familiar, assim como a motivação do próprio aprendiz e reforços positivos ${ }^{18,19}$.

Alguns trabalhos ressaltam a importância das habilidades motoras para o desempenho em tarefas escolares, visto que deficiências nessas habilidades podem gerar atrasos no âmbito da aprendizagem geral ${ }^{20}$. Segundo Rosa Neto, Santos, Xavier e Amaro (2010) existe uma estreita relação entre aquilo que a criança é capaz de aprender (cognitivo) e aquilo que ela é capaz de realizar (motor) ${ }^{21}$. Portanto, o acompanhamento da aptidão motora de crianças em idade escolar cons- 
titui atitude preventiva para os profissionais envolvidos com a aprendizagem.

É imprescindível que a criança, antes de iniciar a sistematização do processo de alfabetização, adquira determinados conceitos ou habilidades que irão permitir e facilitar a aprendizagem da leitura e da escrita ${ }^{14}$.

No presente estudo pode-se verificar a existência de relação entre a habilidade de integração visuo-motora e as habilidades de leitura, escrita e aritmética.

\section{REFERÊNCIAS}

1. Rosa Neto F. Manual de avaliação motora. Porto Alegre: Artmed Editora; 2002. 136p.

2. Sanghavi R, Kelkar R. Visual-motor integration and learning disabled children. The indian journal of occupational therapy. 2005; 37(2):33-8.

3. Ajuriaguerra J et all. A escrita infantil: evolução e dificuldades. Porto Alegre: Artes Médicas; 1988. 301p.

4. Beery KE, Beery NA. The BeeryBuktenica Developmental Test of Visual-Motor Integragion. 6.ed. Texas: Pearson; 2010. 209p.

5. Pinelli Júnior B. Adaptação do Teste do Desenvolvimento da Integração Viso-Motora (VMI) para uso no Brasil [dissertação]. Brasília: Instituto de Psicologia da Universidade de Brasília; $1990.184 p$.

6. Oliveira CR, Rodrigues JC, Fonseca RP. O uso de instrumentos neuropsicológicos na avaliação de dificuldades de aprendizagem. Revista de Psicopedagogia. 2009; 26(79):65-76.

7. Stein LM. Teste de desempenho escolar: manual para aplicação e interpretação. São Paulo: Casa do Psicólogo; 1994. 31p.

8. Kulp MT. Relationship between visual motor integration skill and academic performance in kindergarten through third grade. Optometry and vision science. 1999; 76(3):159-63.

9. Daly CJ, Kelley GT, Krauss A. Relationship between visual-motor integration and handwriting skills of children in kindergarten: a modified replicati-
Também foi identificada a relação entre a habilidade de percepção visual e a habilidade de leitura, bem como a relação entre a habilidade de coordenação motora e a habilidade de escrita com letra cursiva.

Os resultados deste estudo chamam a atenção para a influência das habilidades de integração visuo-motora, percepção visual e coordenação motora sobre o desempenho escolar em atividades de leitura, aritmética e escrita, em especial com letra cursiva.

on study. American journal of occupational therapy. 2003; 57(4):459-62.

10. Nogueira LA, Carvalho LA, Pessanha F C, Lima SCL. A psicomotricidade na prevenção das dificuldades no processo de alfabetização e letramento. Perspectivas online. 2007; 1(2): 928.

11. Oliveira RT, Lopes LG, Magalhães LC. A relação entre integração visuomotora e escrita em crianças de 6 e 7 anos de idade. Temas sobre desenvolvimento. 2004 ; 13(75): 5-13.

12. Capellini AS, Coppede AC, Vale TR. Função motora fina de escolares com dislexia, distúrbio e dificuldades de aprendizagem. Pró-fono revista de atualização científica. 2010; 22(3): 201-8.

13. Feder KP, Majnemer A. Handwriting development, competency and intervention. Developmental Medicine Child Neurology. 2007; 49(4):312-7.

14. Silva $A B$, Borges PFB. A importância da psicomotricidade na educação infantil. Revista de pedagogia perspectivas em educação. 2008;3(1):1-20.

15. Capellini AS, Souza AV. Avaliação da função motora fina, sensorial e perceptiva em escolares com dislexia do desenvolvimento. In: Sennyey $A L$, Capovilla FC, Montiel JM. Transtornos de aprendizagem: da avaliação à reabilitação. São Paulo: Artes Médicas; 2008. p. 55-64.

16. Pelitero TM, Manfredi AKS, Schneck APC. Avaliação das habilidades auditivas em crianças com alterações de aprendizagem. Revista CEFAC. 2010; 12(4): 662-70. 
17. Lucca AS, Mancine MS, Dell'agli BAV. Dificuldade de aprendizagem: contribuições da avaliação neuropsicológica. Revista Científica UNIFAE. 2008; 2(1): 32-42.

18. Felippe ACN, Colafêmina JF. Avaliação simplificada do processamento auditivo e o desempenho em tarefas de leitura- escrita. Pró-Fono Revista de Atualização Científica. 2002; 4(2): 225-34.

19. Siqueira CM, Gurgel-Giannetti J. Mau desempenho escolar: uma visão atual, Revista Associação Médica Brasileira. 2011;57(1):78-87.

20. Medina-Papst J, Marques I. Avaliação do desenvolvimento motor de crianças com dificuldades de aprendizagem. Revista Brasileira de Cineantropometria e Desempenho Humano. $2010 ; 12(1): 36-42$.

21. Rosa Neto F, Santos APM, Xavier RFC, Amaro KN. A importância da avaliação motora em escolares: análise da confiabilidade da Escala de Desenvolvimento Motor. Revista brasileira de Cineantropometria e desempenho humano.2010; 12(6): 422-27. 